\title{
A INCOMUM ASSOCIAÇÃO DE PEIXES E CARANGUEJOS DA FORMAÇÃO ROMUALDO, APTIANO-ALBIANO DA BACIA SEDIMENTAR DO ARARIPE, NE DO BRASIL
}

\author{
LUDMILA ALVES CADEIRA DO PRADO ID, \\ GABRIEL LEVI BARBOSA LOPES (D) \\ Departamento de Geologia, Centro de Tecnologia e Geociências, \\ Universidade Federal de Pernambuco, UFPE, Recife, PE, Brasil. \\ prado.lac@gmail.com,gabriellbll@gmail.com
}

\section{PRISCILLA ALBUQUERQUE PEREIRA (D)}

Departamento de Biologia, Universidade Federal Rural de Pernambuco, UFRPE, Recife, PE, Brasil. prizinha.albuquerque@gmail.com

\section{RILDA VERÔNICA CARDOSO DE ARARIPE (D Departamento de Geologia, Centro de Tecnologia e Geociências, Universidade Federal de Pernambuco, UFPE, Recife, PE, Brasil. rildacardoso@gmail.com}

\section{DAVID HOLANDA DE OLIVEIRA (D}

Departamento de Biociências, Centro de Ciências Agrárias, Universidade Federal da Paraíba, UFPB, Areia, PB, Brasil. david@cca.ufpb.br

\author{
FLAVIA AZEVEDO PEDROSA LEMOS D, LUIZ RICARDO DA SILVA LOBO \\ DO NASCIMENTO D, MARIA EMÍLIA TRAVASSOS RIOS TOMÉ (D) \\ \& ALCINA MAGNÓLIA FRANCA BARRETO (D) \\ Departamento de Geologia, Centro de Tecnologia e Geociências, \\ Universidade Federal de Pernambuco, UFPE, Recife, PE, Brasil. \\ flaviapedrosa.geo@gmail.com, exinarico@gmail.com, \\ maria.emilia.tome@gmail.com, alcinabarreto@gmail.com
}

\begin{abstract}
The ununsual association of fish and crabs from Romualdo Formation, Aptian-Albian of the Araripe sedimentary Basin, NE Brazil. In a study and collection of fossils from the Romualdo Formation in "Araripe pernambucano", an association of Vinctifer comptoni and Rhacolepis buccalis with eubrachyurans and orithopsids crabs was observed in four calcareous nodules. Associations were discussed based on ecological and taphonomic hypotheses. It is noted from the degree of articulation of the organisms, that either the death or the availability of crab moults in the sediment possibly occurred before the death of the fish. In addition, the fish do not have teething consistent with feeding on crabs. Thus, this association would occur due to fossilization processes and not due to ecological interactions. The study also emphasized systematic and paleogeographic aspects of the groups. Romualdocarcinus salesi is probably related to the "higher" true crabs (Eubrachyura), a hypothesis already raised in previous works, due to the preservation of its dorsal carapace next to a pleon of subquadrangular shape similar to those seen in this group. The occurrence of Exucarcinus gonzagai associated with fish, in concretions in the shales, can raise the question of E. gonzagai and Araripecarcinus ferreirai species as synonyms. The data obtained with the survey of the paleogeographic distribution of fish and crabs in geological formations of correlated age corroborates the hypothesis of the Tethyan marine influence in Araripe.
\end{abstract}

Keywords: ichthyolites, decapods, paleoecology, taphonomy, Santana Group, Cretaceous. 
RESUMO - Em estudo e coleta de fósseis da Formação Romualdo no Araripe pernambucano, observou-se, em quatro nódulos calcários, uma associação de Vinctifer comptoni e Rhacolepis buccalis com caranguejos eubraquiúras e oritopsídeos. Foram discutidas as associações a partir das hipóteses ecológicas e tafonômicas. Nota-se a partir do grau de articulação dos organismos, que ou a morte ou a disponibilização de mudas dos caranguejos no sedimento possivelmente se deram previamente a morte dos peixes. Além disso, os peixes não possuem dentição condizente com alimentação a partir de caranguejos. Assim, esta associação se daria devido aos processos de fossilização e não devido a interações ecológicas. O estudo também enfatizou aspectos sistemáticos e paleogeográficos dos grupos. Romualdocarcinus salesi estaria possivelmente relacionado aos caranguejos verdadeiros mais "avançados" (Eubrachyura), hipótese já levantada em trabalhos anteriores, devido a preservação de sua carapaça dorsal junto a um pléon de formato subquadrangular semelhante aos vistos neste grupo. A ocorrência de Exucarcinus gonzagai associado aos peixes, em concreções nos folhelhos, pode colocar em discussão as espécies E. gonzagai e Araripecarcinus ferreirai como sinonímias. Os dados obtidos com o levantamento da distribuição paleogeográfica de peixes e caranguejos em formações geológicas de idade correlata corroboram com a hipótese da influência marinha tetiana no Araripe.

Palavras-chave: ictiólitos, decápodes, paleoecologia, tafonomia, Grupo Santana, Cretáceo.

\section{INTRODUÇÃO}

A Formação Romualdo (Neumann \& Cabrera, 1999) é um importante jazigo fossilífero da Bacia do Araripe em termos qualitativos e quantitativos (Maisey, 1991). Possui uma fauna diversificada, relacionada às variações ambientais do sistema transgressivo-regressivo, representada por peixes, répteis (pterossauros, dinossauros, crocodilos, tartarugas), equinoides irregulares, moluscos (gastrópodes e bivalves), crustáceos (ostracodes, caranguejos, camarões), plantas (pteridófitas, gimnospermas e angiospermas), palinomorfos e foraminíferos (Beurlen, 1963; 1964; 1966; Silva-Santos \& Valença, 1968; Arai \& Coimbra, 1990; Maisey, 1991; Kellner, 2002; Oliveira, 2007; Bruno \& Hessel, 2006; Lima et al., 2012; Pinheiro et al., 2014; Pereira et al., 2017; Prado et al., 2018a, b).

A paleoictiofauna da Formação Romualdo tem sido estudada desde o século XIX, com os trabalhos pioneiros em taxonomia de Louis Agassiz em 1841. Ela é composta por mais de 40 espécies, com representantes de Chondrichthyes e Osteichthyes (Actinopteryigii e Sarcopterygii), de acordo com Silva-Santos \& Valença (1968), Brito et al. (2013), Polck et al. (2015), entre outros. Dentre estes, os táxons citados como os mais comuns são Vinctifer comptoni Agassiz, 1841, Rhacolepis buccalis Agassiz, 1841 e Tharrhias araripis Jordan \& Branner, 1908. Além de trabalhos descritivos, a paleoictiofauna também é foco de estudos paleoecológicos desde a década de 1960, com destaque para Silva-Santos \& Valença (1968), e mais recentemente, Maisey (1994) e Maisey \& Carvalho (1995).

Os caranguejos da Formação Romualdo são representados até o presente pelas espécies Araripecarcinus ferreirai MartinsNeto, 1987 (Necrocarcinidae?) e Exucarcinus gonzagai Prado e Luque, 2018 (Orithopsidae), ambos pertencentes a seção Raninoida (Karasawa et al., 2014), e Romualdocarcinus salesi Prado e Luque, 2018 (Eubrachyura?). Enquanto E. gonzagai e $R$. salesi são reconhecidos por dezenas de carapaças dorsais associadas a moluscos e equinoides, $A$. ferreirai é representado por um único espécime com a porção ventral da carapaça preservada associado ao peixe Vinctifer comptoni (Martins-Neto, 1987; Luque, 2015; Prado et al., 2018b).

Desde então, nenhuma outra associação de peixe e caranguejo nas concreções calcárias da Formação Romualdo foi divulgada, dada a raridade da ocorrência ou a não percepção dos decápodes, dado seu tamanho diminuto nas concreções. O presente trabalho visa discorrer sobre novos exemplares que caracterizam essa associação, bem como as possíveis interações ecológicas e alterações tafonômicas envolvidas no processo de preservação.

\section{GEOLOGIA DA BACIA E CONTEXTO PALEOGEOGRÁFICO}

Eventos tectônicos responsáveis pela ruptura e fragmentação do Supercontinente Gondwana durante o Mesozoico e a consequente evolução do oceano Atlântico sul, foram responsáveis pela reativação de falhas pré-cambrianas no interior do nordeste brasileiro que influenciaram diretamente na estruturação interna das bacias intracratônicas, dentre elas, a Bacia do Araripe (Matos, 1992).

A Bacia do Araripe localiza-se entre os estados de Pernambuco, Piauí e Ceará e registra as sequências estratigráficas pré-rifte, rifte, pós-rifte I e II (Figura 1), representadas, sobretudo, por estratos lacustres e fluviais (Ponte e Ponte Filho, 1996; Assine, 2007; Marques et al., 2014). Na sequência pós-rifte I, encontra-se a Formação Romualdo, uma unidade composta por conglomerados, arenitos, siltitos, calcários e, principalmente por folhelhos que registram a ingressão marinha que alcançou o Nordeste do Brasil durante o Eocretáceo.

Do ponto de vista paleontológico, a Formação Romualdo destaca-se mundialmente pela diversidade e excepcional preservação dos fósseis em concreções calcárias, principalmente peixes (Konservat-Lagerstätten, Martill, 1988; Maisey, 1991). Mais recentemente, fósseis de invertebrados marinhos, tais como foraminíferos, moluscos, equinodermas e decápodes, têm chamado atenção por contribuir com o estudo paleoambiental e paleogeográfico do sentido da transgressão marinha no Araripe, assunto ainda controverso (Beurlen, 1963; 1966; Arai, 2014; Pereira et al., 2017; Prado et al., 2018a, b; Araripe et al., 2019; Melo et al., 2020).

A presença de diversos grupos taxonômicos com afinidade tetiana nas bacias do nordeste seria uma das evidências da ingressão a partir do Mar de Tetis (Beurlen, 1963; 1966; Arai et al., 1994; Arai, 2014; Prado et al., 2015). Segundo 


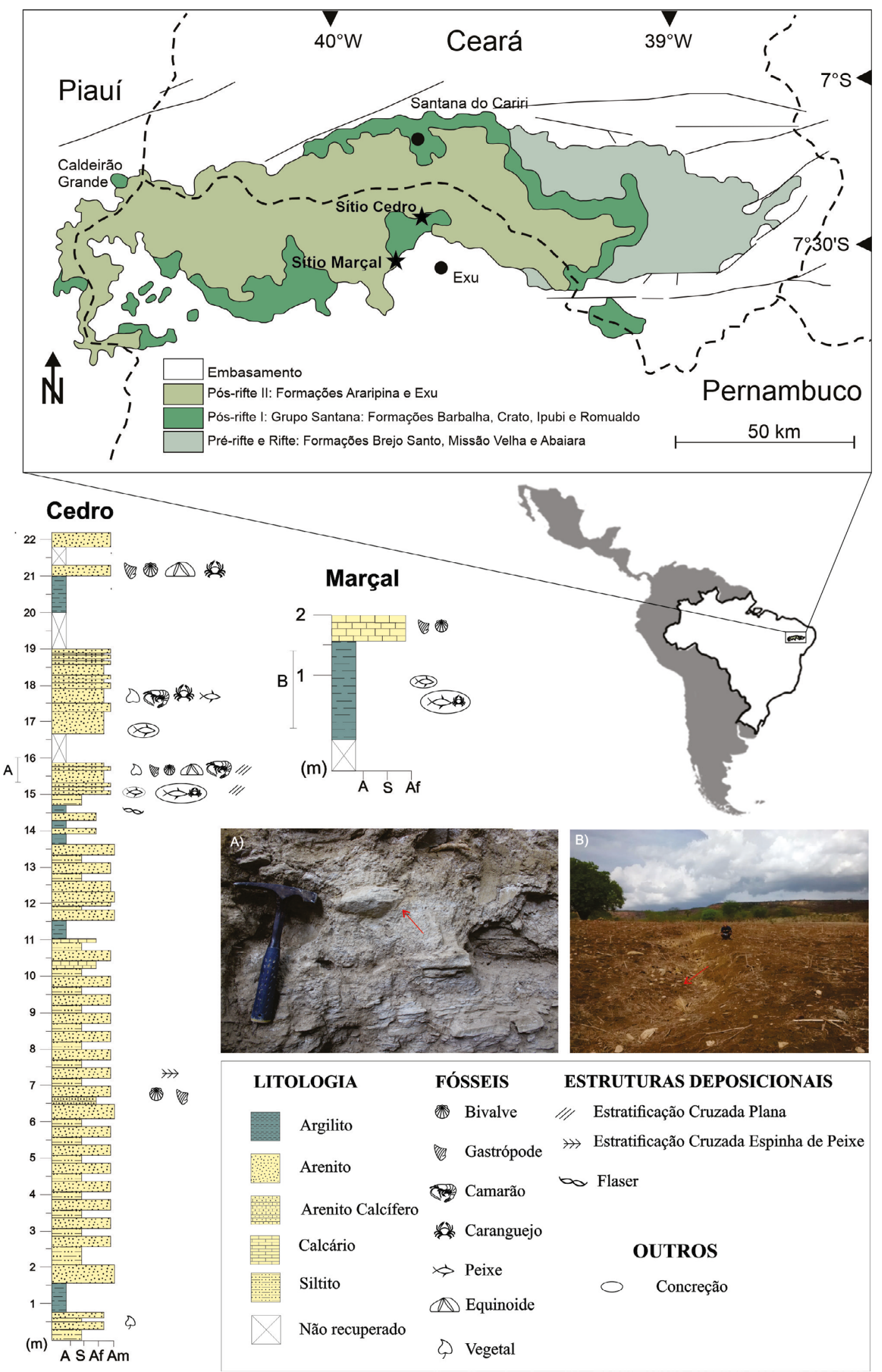

Figura 1. Localização das áreas de ocorrência da associação de peixes e caranguejos em concreções calcárias da Formação Romualdo e sua distribuição nos perfis geológicos do município de Exu, Estado de Pernambuco. As setas indicam os ictiólitos. A, Sítio Cedro; B, Sítio Marçal.

Figure 1. Location of areas of occurrence of the association of fish and crabs in limestone concretions of the Romualdo Formation and their distribution in the geological profiles of the municipality of Exu, State of Pernambuco. The arrows indicate the ichthyolites. A, Cedro Site; B, Marçal Site. 
Arai (2014), o mar alcançaria as bacias do Araripe e SergipeAlagoas a partir da Bacia do Parnaíba, onde a ecozona de dinoflagelados tetianos (gênero Subtilisphaera) é mais proeminente. Em uma direção oposta, a ingressão via Bacia Sergipe-Alagoas encontra suporte nas paleocorrentes fluviais medidas nas formações Barbalha (pós-rifte I) e Marizal (Bacia do Tucano), que indicam paleofluxo para o sul e sudeste (Assine et al., 2014; 2016). Outras hipóteses levantadas abrangem a conexão entre as bacias do Araripe e Potiguar, baseada em falhas com direção SW (Lima, 1978) e a junção de todas essas bacias num único seaway (e.g. Mabesoone et al., 1999; Valença et al., 2003).

\section{MATERIAL E MÉTODOS}

Em levantamento de dados estratigráficos e coletas de fósseis de vertebrados e invertebrados da Formação Romualdo no Araripe pernambucano, quatro concreções calcárias com fósseis de peixes foram encontradas associadas a caranguejos (Figuras 2-5) nos sítios Marçal e Cedro, município de Exu, Pernambuco (PE) (Figura 1). Aproximadamente 300 concreções foram analisadas contendo os peixes dos gêneros Vinctifer, Rhacolepis, Calamopleurus, Cladocyclus, Neoproscinetes, Paraelops, Tharrhias, Notelops, Araripelepidotes, Brannerion, e Beurlenichthys, plantas (Brachyphyllum), tartarugas das espécies Araripemys barretoi Price, 1973, e Cearachelys placidoi Gaffney, Almeida Campos \& Hirayama, 2001, e coprólitos. Os nódulos foram abertos de forma mecânica com martelo geológico. Os peixes foram identificados a partir da descrição e comparações com a bibliografia de Maisey (1991) e Polck et al. (2015). Os caranguejos foram preparados com auxílio de agulhas e micro retífica a fim de expor a carapaça e facilitar a identificação taxonômica. A identificação dos espécimes foi baseada na análise das feições dorsais da carapaça. O material coletado encontra-se depositado na coleção de Paleontologia do Departamento de Geologia, Centro de Tecnologia e Geociências da Universidade Federal de Pernambuco sob os números de tombo: DGEO-CTG-UFPE-8247, DGEOCTG-UFPE-8302, DGEO-CTG-UFPE-8311 e DGEO-CTGUFPE-8748.

\section{RESULTADOS E DISCUSSÃO}

As espécies de caranguejos identificadas nas concreções correspondem às já descritas na Formação Romualdo: Exucarcinus gonzagai e Romualdocarcinus salesi (Prado et al., 2018b). Essas carapaças estavam associadas aos peixes das espécies Vinctifer comptoni (DGEO-CTG-UFPE-8748) e Rhacolepis buccalis (DGEO-CTG-UFPE-8302 e DGEOCTG-UFPE-8247), além de um espécime indeterminado (DGEO-CTG-UFPE-8311). Neste último não há preservação de elementos craniais nem nadadeira caudal que permitam a identificação (Figuras 2-6). Com exceção de $V$. comptoni coletado no sítio Marçal, todos os outros materiais foram coletados no sítio Cedro, ambos localizados no município de Exu, PE (Figura 1). Segundo Polck et al. (2015), estas espécies de peixes seriam duas das três mais comuns e abundantes da Bacia do Araripe. Em Exu, a afirmação também é verdadeira, sendo $V$. comptoni o táxon mais comum da fauna de vertebrados do município, seguido por $R$. buccalis (Duque \& Barreto, 2018).

Para tentar explicar a associação entre caranguejos e peixes presente nos nódulos calcários da Formação Romualdo, foram levantadas e discutidas algumas possibilidades paleoecológicas e tafonômicas descritas a seguir:

\section{Predação}

Embora Vinctifer comptoni e Rhacolepis buccalis apresentassem uma morfologia fusiforme e fossem considerados peixes pelágicos, os seus hábitos alimentares eram bem diferentes, evidenciados pela morfologia dos dentes e/ou pelo conteúdo estomacal preservado durante o processo de fossilização.

A dentição de Rhacolepis buccalis era composta por dentes pequenos e pontiagudos, sendo possível classificá-la como uma dentição do tipo viliforme (Bemvenuti \& Fisher, 2010). Esse tipo de dentição mostra especificidade para se alimentar de pequenos peixes (Santanichthys diasii Silva Santos, 1958, Rhacolepis buccalis jovens) e camarões (Paleomattea deliciosa Maisey e Carvalho, 1995) como foi relatado por Maisey (1994) e Maisey \& Carvalho (1995) por meio de análise do seu conteúdo estomacal.

Vinctifer comptoni, por sua vez, apresenta uma dentição reduzida formando uma pequena serrilha, e embora ele já tenha sido considerado um predador piscívoro da Formação Romualdo (Silva-Santos \& Valença, 1968), é muito provável que $V$. comptoni fosse uma espécie filtradora, devido ao tamanho dos dentes e ao aparelho branquial bem desenvolvido (Maisey, 1994).

Muitos peixes possuem hábito oportunista, como por exemplo alguns peixes do gênero Serrasalmus (Pompeu, 1999; Costa et al., 2005), se alimentando de outros recursos além de sua fonte primária de alimentação. No entanto não é possível aplicar o hábito oportunismo para a relação peixe/ caranguejo descrita nesse trabalho, visto que no conteúdo estomacal de ambas as espécies, analisado previamente por Maisey (1994), Wilby \& Martill (1992), e Brito (1997), não foi observado nenhuma estrutura relacionada a caranguejos.

Não foram observadas nenhuma marca de mordida nas carapaças analisadas. Bishop (1972a, 1978) analisou fraturas e marcas de mordidas pequenas, arredondadas e espaçadas em uma carapaça da espécie Raninella oaheensi Bishop, 1978 do Cretáceo Superior da Dakota do Sul, dentre mais de 400 espécimes de decápodes da coleção estudada, inferindo predação por peixes. De fato, evidências de predação em caranguejos são raras no registro fóssil, uma vez que são facilmente destruídas quando o predador obtém sucesso e se alimenta da presa. Logo, evidências de alimentação de decápodes são mais comuns em conteúdos estomacais (Klompmaker et al., 2013). 

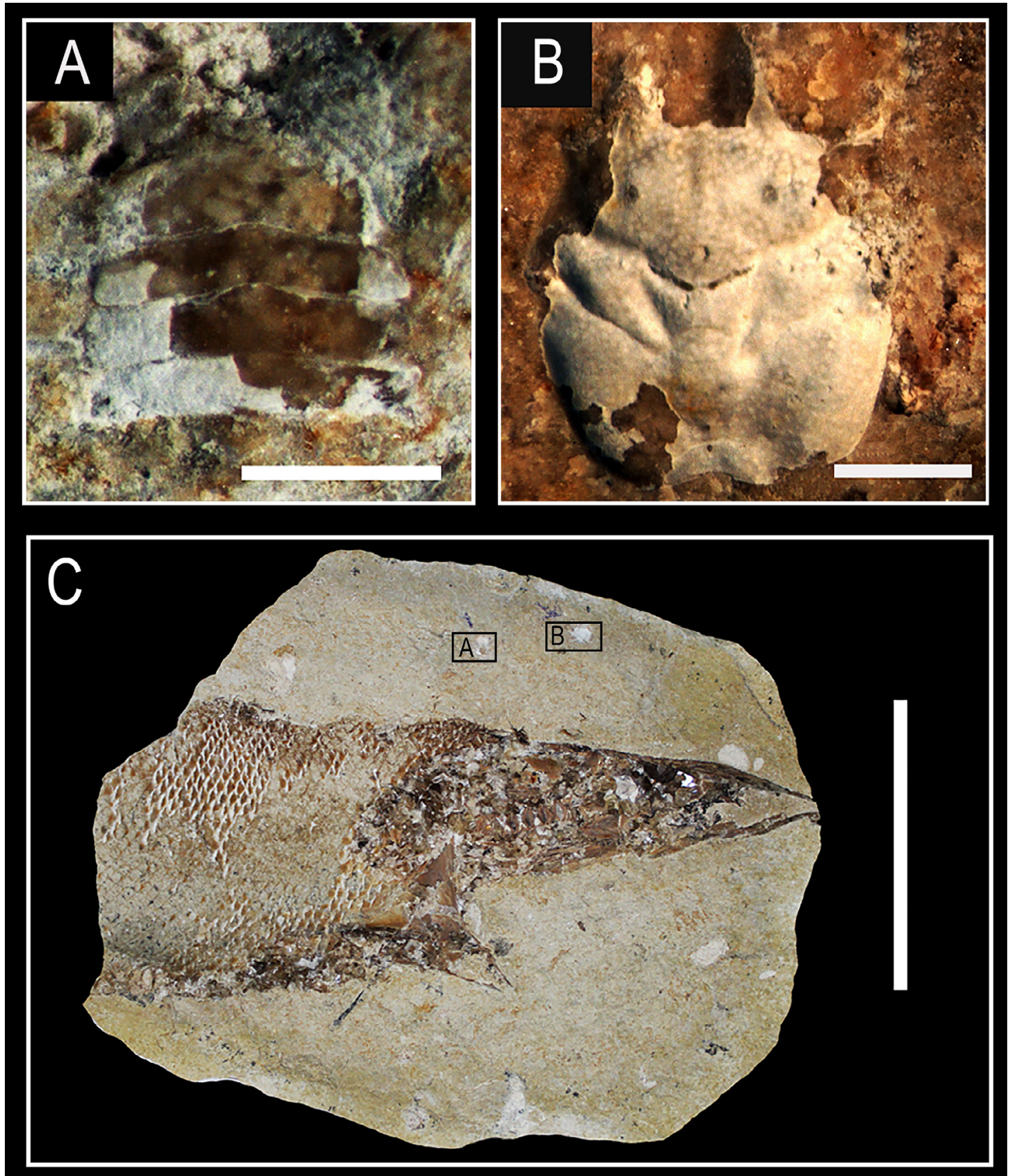

Figura 2. Associação de peixe e caranguejos em concreções calcárias da Formação Romualdo (DGEO-CTG-UFPE -8247). A, pléon de Romualdocarcinus salesi (?); B, R. salesi; $\mathbf{C}$, Rhacolepis buccalis. Escalas: A-B $=1 \mathrm{~mm} ; \mathrm{C}=5 \mathrm{~mm}$.

Figure 2. Association of fish and crabs in calcareous concretions of the Romualdo Formation (DGEO-CTG-UFPE -8247). A, pleon of Romualdocarcinus salesi (?); B, R. salesi; $\mathbf{C}$, Rhacolepis buccalis. Scale bars: A-B $=1 \mathrm{~mm} ; \mathrm{C}=5 \mathrm{~cm}$.

Baseado nas características morfológicas de ambas as espécies, é possível inferir que embora haja uma associação fossilífera entre caranguejos e peixes, era pouco provável que os caranguejos fossem fonte de alimento para esses peixes.

\section{Instabilidade ambiental}

A comum presença de peixes em concreções da Formação Romualdo poderia resultar de eventos de mortandade em massa causada por variações ambientais, tais como flutuações de salinidade, taxa de oxigênio e temperatura ou até mesmo proliferação de algas (Martill, 1988; Martill et al., 2008). A instabilidade ambiental facilmente causaria a morte de peixes e bentos mais sensíveis a estas mudanças. Contudo, não parece plausível afirmar que ambos os organismos teriam morrido a partir de um mesmo evento de mortandade, já que peixes e caranguejos são encontrados em diferentes estágios de desarticulação. 

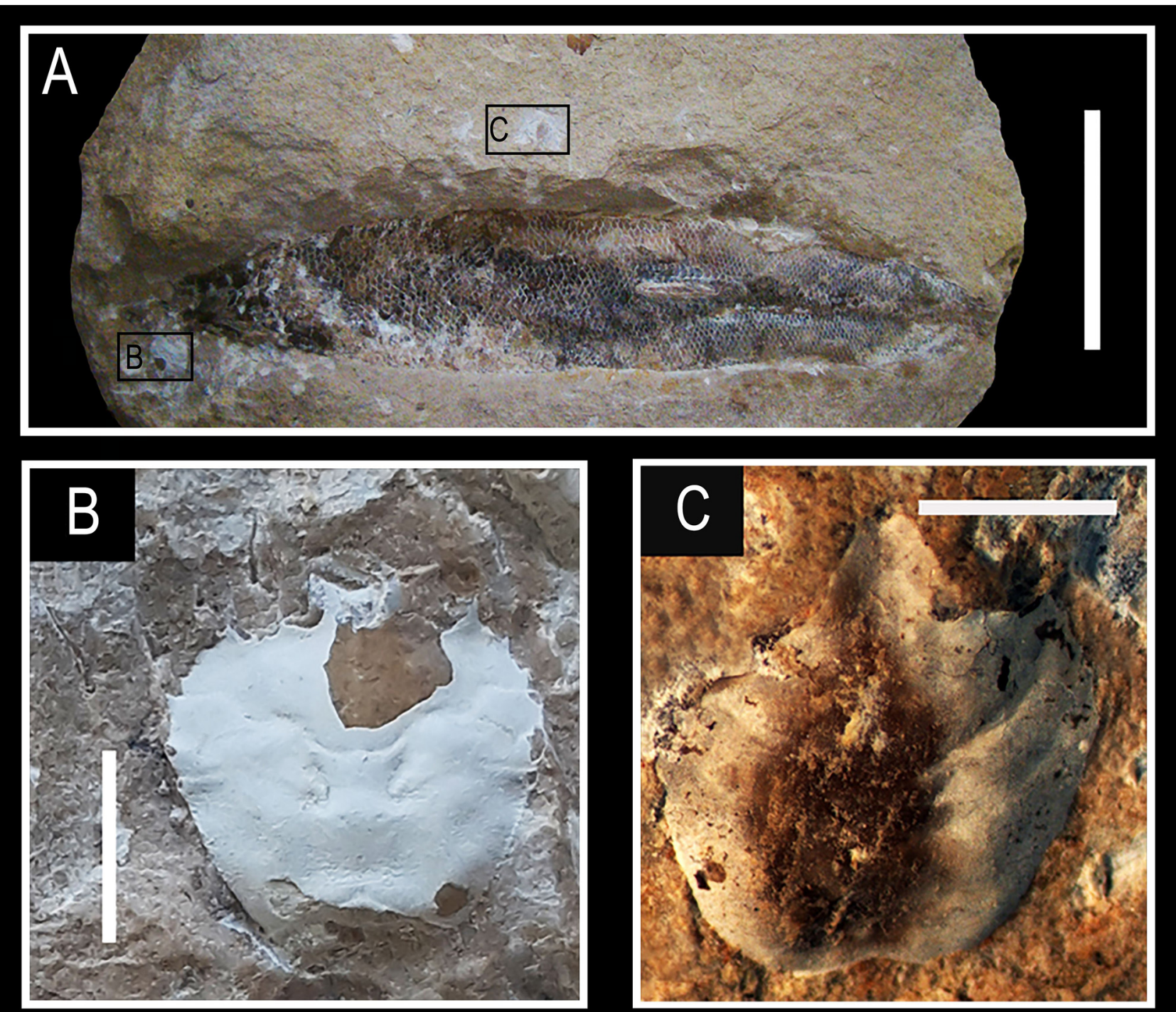

Figura 3. Associação de peixe e caranguejos em concreções calcárias da Formação Romualdo (DGEO-CTG-UFPE-8302). A, Rhacolepis buccalis; B-C, Exucarcinus gonzagai. Escalas: $\mathrm{A}=5 \mathrm{~cm} ; \mathrm{B}=5 \mathrm{~mm} ; \mathrm{C}=2 \mathrm{~mm}$.

Figure 3. Association of fish and crabs in calcareous concretions of the Romualdo Formation (DGEO-CTG-UFPE-8302). A, Rhacolepis buccalis; B-C, Exucarcinus gonzagai. Scale bars: $\mathrm{A}=5 \mathrm{~cm} ; \mathrm{B}=5 \mathrm{~mm} ; \mathrm{C}=2 \mathrm{~mm}$.

Em todas as amostras analisadas, os caranguejos encontram-se desarticulados, tendo havido o desligamento dos pereópodes do cefalotórax, mostrando que um pequeno intervalo de tempo provavelmente decorreu desde a sua morte ao soterramento final. Alguns autores (Allison, 1986; Plotnick, 1986; Poulicek et al., 1988; Stempien, 2005), baseados em trabalhos tafonômicos atualísticos, sugerem que a quitina presente nos artrópodes é suscetível à decomposição por fungos ou bactérias dentro de duas a quatro semanas, especialmente em condições subaquáticas.

$\mathrm{O}$ mesmo não se observa nos peixes, que se apresentam articulados, com preservação de partes moles e com a integridade da forma preservada. Segundo Martill (1988, 1989), a boa preservação na maioria dos fósseis nas concreções da Formação Romualdo sugere que a precipitação do carbonato teria ocorrido logo após ou até antes do soterramento, permitindo a preservação de tecidos moles como as escamas e os arcos branquiais. Essa preservação quase instantânea foi chamada de "Efeito Medusa" por Martill (1989), em alusão à personagem mitológica Medusa que transformava seus observadores em pedras por toda eternidade. É importante destacar que os peixes alcançaram o sedimento logo após a morte. Segundo Martill (1988), peixes da Formação Romualdo também poderiam flutuar por um período prolongado devido a produção de gases intestinais no processo de decomposição. Entretanto, não foi observado nos fósseis aqui estudados nenhuma evidência de rompimento de tecidos que justificassem a ocorrência desse processo.

Presume-se que os caranguejos teriam mortes anteriores a dos peixes e estariam preservados juntos como produto de uma time-averaging. Tal assertiva baseia-se no estágio de desarticulação dos organismos: peixes seriam rapidamente 

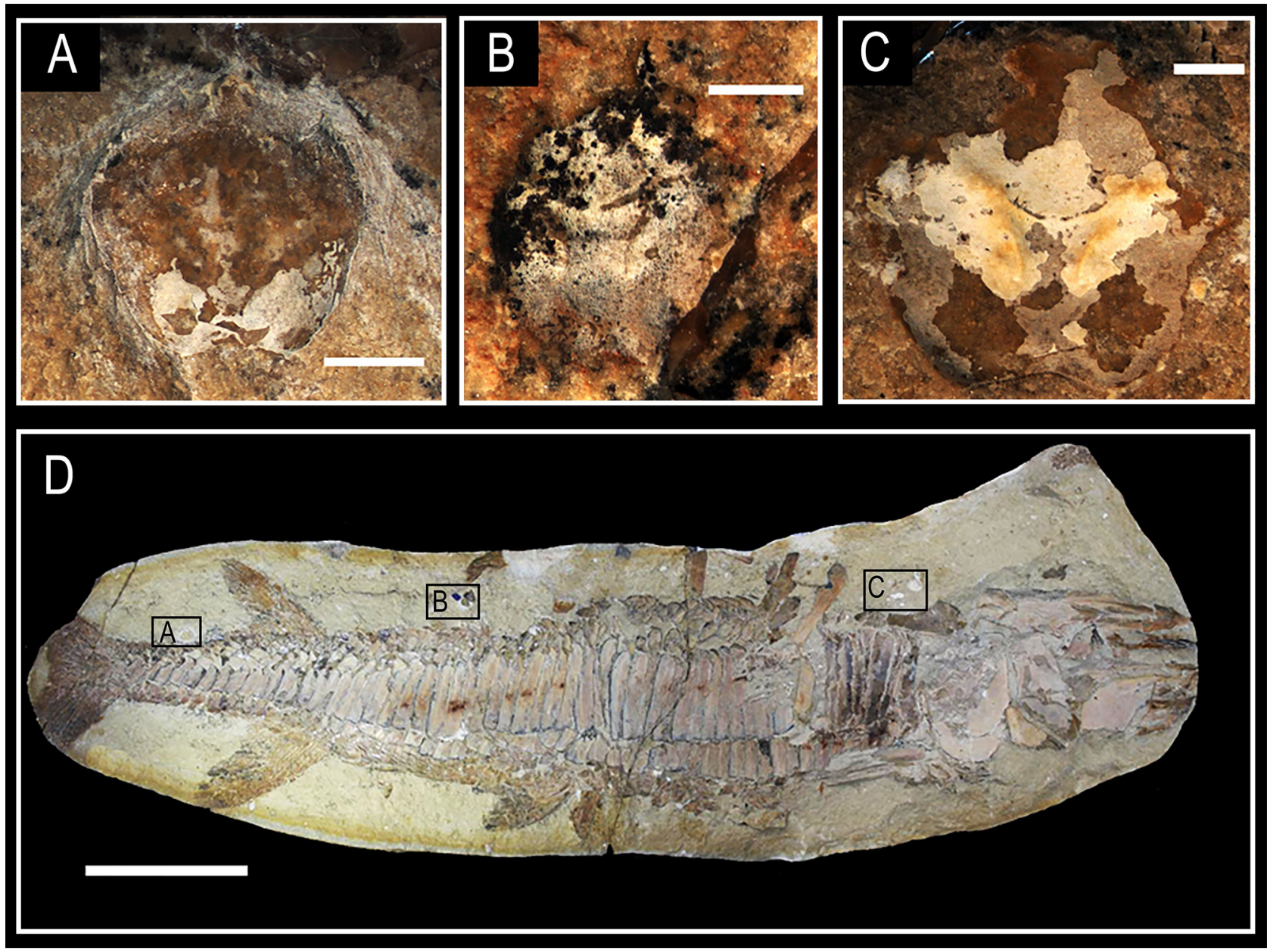

Figura 4. Associação de peixe e caranguejos em concreções calcárias da Formação Romualdo (DGEO-CTG-UFPE- 8748). A, Exucarcinus gonzagai; B-C, caranguejos indeterminados; $\mathbf{D}$, Vinctifer comptoni. Escalas: A, $\mathrm{C}=2 \mathrm{~mm} ; \mathrm{B}=1 \mathrm{~mm} ; \mathrm{D}=5 \mathrm{~cm}$.

Figure 4. Association of fish and crabs in calcareous concretions of the Romualdo Formation (DGEO-CTG-UFPE-8748). A, Exucarcinus gonzagai; B-C, indeterminate crabs; D, Vinctifer comptoni. Scale bars: A, C $=2 \mathrm{~mm} ; \mathrm{B}=1 \mathrm{~mm} ; \mathrm{D}=5 \mathrm{~cm}$.

soterrados ainda articulados logo após a morte com uma rápida ou instantânea precipitação carbonática e caranguejos estariam disponíveis no sedimento tempo suficiente para haver desarticulação, ou seja, apresentariam um maior tempo de residência na zona tafonomicamente ativa (ZTA).

\section{Necrofagia/Carnivoria}

De uma forma geral, caranguejos têm uma dieta onívora, podendo se alimentar de outros invertebrados, algas, pequenos peixes e matéria orgânica em decomposição. Segundo Guinot et al. (2008), raninoides fósseis, tais como Exucarcinus gonzagai, seriam carnívoros e/ou necrófagos baseado na morfologia dos seus quelípedes. Espécies atuais do grupo, ex. Raninoides lamarcki A. Milne-Edwards \& Bouvier, 1923, ainda apresentam esse mesmo hábito alimentar.

No entanto, dificilmente essa interação ecológica seria encontrada no registro fóssil, uma vez que marcas de carnivoria/necrofagia causadas por caranguejos em tecidos de vertebrados raramente seriam preservadas ou notadas. Além disso, como citado acima, é muito mais provável que os organismos não tenham interagido ecologicamente, e a presença dos peixes no local de sedimentação tenha se dado após a morte dos caranguejos.

\section{Ecdise}

Os espécimes identificados ainda poderiam corresponder a mudas e não a restos corporais. A ecdise em caranguejos se dá através da abertura da sutura pleural, com a região pterigostomial conectada ao esterno. A preservação dos corpos é confirmada com a presença da região pterigostomial intacta e conectada ao resto da carapaça (Bishop, 1972b; 1986). Nas associações aqui estudadas apenas a porção dorsal da carapaça é vista numa preservação bidimensional, não sendo possível afirmar se correspondem a mudas. A exemplo de Klompmaker et al. (2016), os espécimes também poderiam corresponder a restos corporais se ou as carapaças ou a região pterigostomial de ambos os lados da carapaça tivessem se desarticulado do resto do corpo e sofrido transporte.

Assim, o pensamento mais parcimonioso seria considerar que as tanatocenoses em questão são formadas por mudas e/ 

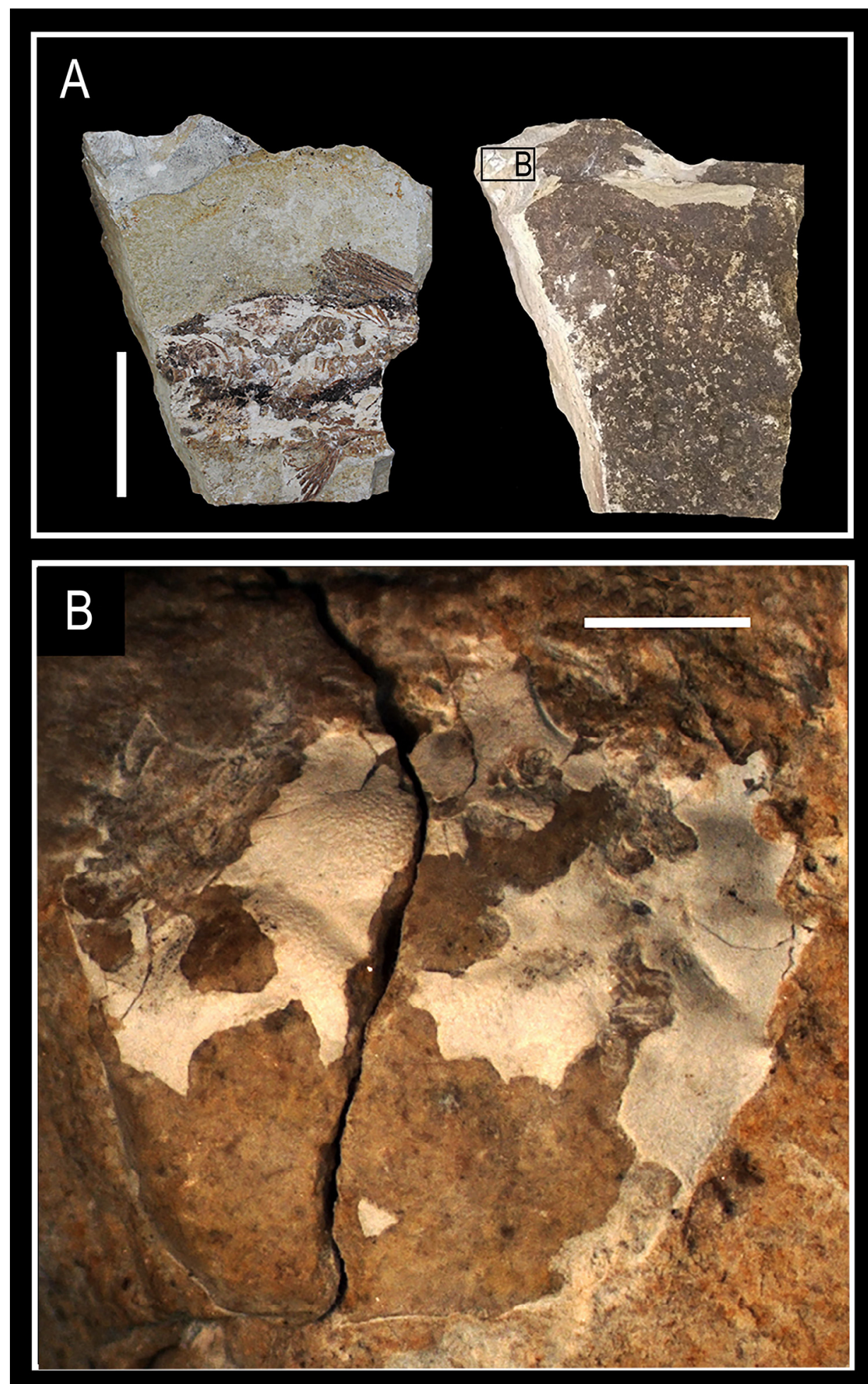

Figura 5. Associação de peixe e caranguejos em concreções calcárias da Formação Romualdo (DGEO-CTG-UFPE-8311). A, peixe indeterminado (parte interna e externa do ictiólito); B, Romualdocarcinus salesi. Escalas: $\mathrm{A}=5 \mathrm{~cm} ; \mathrm{B}=2 \mathrm{~mm}$.

Figure 5. Association of fish and crabs in calcareous concretions of the Romualdo Formation (DGEO-CTG-UFPE-8311). A, indeterminate fish (internal and external part of ichthyolite); B, Romualdocarcinus salesi. Scale bars: $\mathrm{A}=5 \mathrm{~cm} ; \mathrm{B}=2 \mathrm{~mm}$.

ou corpos de caranguejos depositados antes dos peixes no sedimento. Uma vez juntos, caranguejos e peixes seriam abruptamente soterrados em uma mesma associação fossilífera.

\section{Romualdocarcinus salesi, um Eubrachyura?}

Em Cedro, um pléon de caranguejo isolado foi encontrado levemente deslocado da carapaça dorsal de Romualdocarcinus salesi dentro da concreção com o peixe Rhacolepis buccalis 

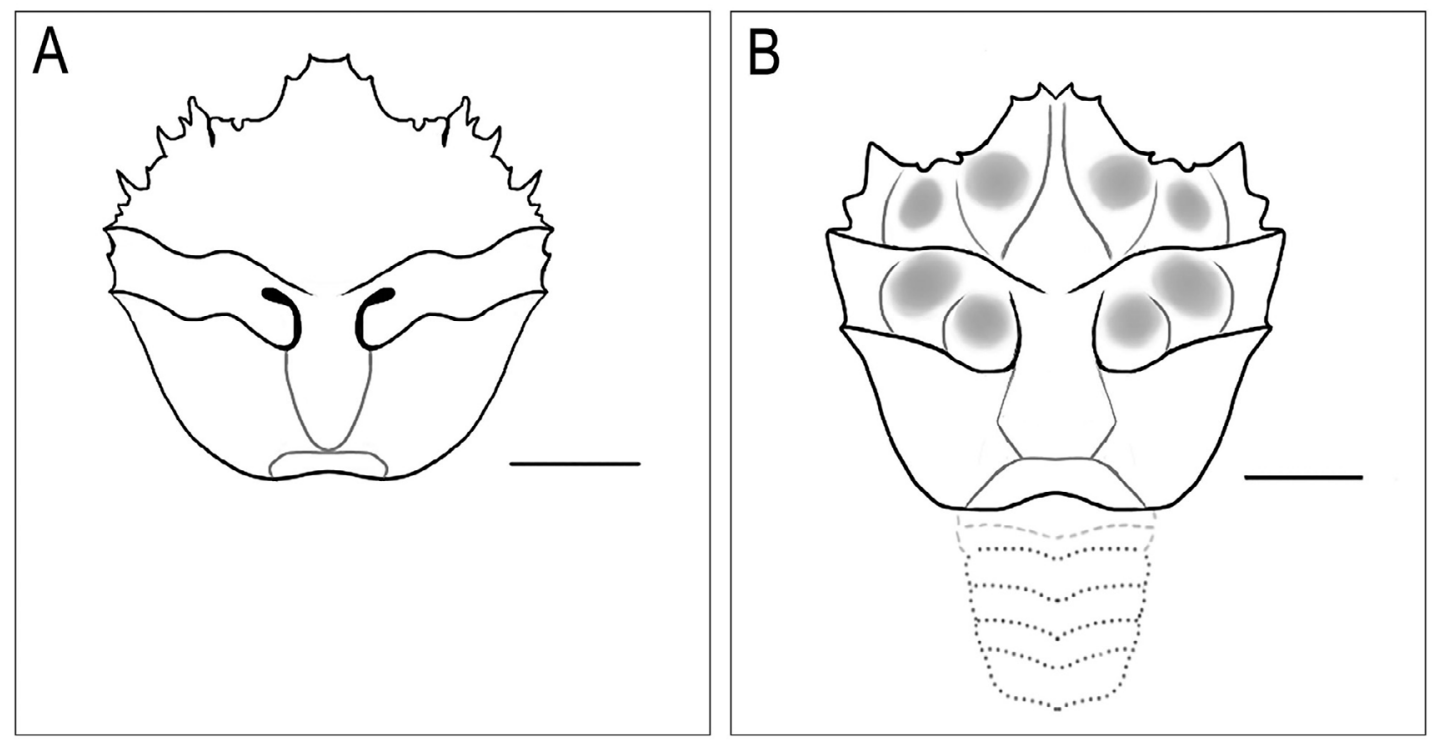

Figura 6. Reconstrução das espécies de caranguejos. A, carapaça dorsal de Exucarcinus gonzagai; B, carapaça dorsal e possível pléon (tracejado) de Romualdocarcinus salesi. Escalas: A $=5 \mathrm{~mm} ; \mathrm{B}=2 \mathrm{~mm}$.

Figure 6. Reconstruction of crab species. A, dorsal carapace of Exucarcinus gonzagai; B, dorsal carapace and possible pleon (dashed) of Romualdocarcinus salesi. Scale bars: $\mathrm{A}=5 \mathrm{~mm} ; \mathrm{B}=2 \mathrm{~mm}$.

(Figuras 2 e 6b). Embora não se possa afirmar que o pléon em questão corresponda $R$. salesi, este não pertenceria aos outros dois caranguejos presentes na Formação Romualdo: Exucarcinus gonzagai (Orithopsidae) e Araripercarcinus ferreirai (Necrocarcinidae?), devido ao seu formato mais alargado; raninoides possuem um pleón estreito característico (Schweitzer et al., 2012).

O caranguejo Romualdocarcinus salesi foi classificado como um possível eubraquiúra da família Eogeryonidae, que incluía até então um único gênero, Eogeryon. Se considerarmos que o pléon isolado seria de R. salesi, esta espécie representaria de fato uma das ocorrências de caranguejos "avançados" mais antigas do mundo.

O pléon é largo, subquadrangular e amplo, possivelmente de uma fêmea (Figuras 2a, 6b), não sendo possível assim compará-lo com aquele de Eogeryon, que é macho; as diferenças podem refletir facilmente dimorfismo sexual. Contudo, comparando o pleón com aqueles de fêmeas de eubraquiúros basais do Cretáceo, são semelhantes, tal como Marocarcinus pasinii Guinot, De Angeli \& Garassino, 2008 (Marocarcinidae).

\section{Araripecarcinus ferreirai versus Exucarcinus gonzagai}

É importante salientar que Prado et al. (2018b) mencionam que Exucarcinus gonzagai (representado pela carapaça dorsal) possivelmente não seria coespecífico a Araripecarcinus ferreirai (caracterizado pela região ventral) pelas diferentes associações faunísticas em que eram encontrados, e que refletiriam por sua vez, diferentes subambientes. Até o momento, E. gonzagai estava associado apenas aos invertebrados no município de Exu, PE, inclusive no sítio Cedro (Prado et al., 2018b). A. ferreirai foi coletado no nível dos peixes (Martins-Neto, 1987) no município de Jardim, CE. A nova ocorrência de E. gonzagai associado aos peixes ascende a discussão, podendo E. gonzagai e $A$. ferreirai serem, portanto, sinonímias.

\section{Inferências paleoambientais}

Os peixes são organismos que ocupam diversos tipos de ambientes, desde rios e lagos até os grandes oceanos, sendo que algumas espécies suportam uma ampla variação de salinidade, mostrando que podem habitar em diversos ambientes ao longo de sua vida.

Os táxons descritos neste trabalho são considerados como espécies que habitavam ambiente marinho (Maisey, 1991; 1994), no entanto, alguns autores sugerem que Rhacolepis buccalis além de ocupar ambiente marinho, poderia também viver em ambientes estuarinos (Silva-Santos \& Valença, 1968; Mabesoone \& Tinoco, 1973; Bruno \& Hessel, 2006). Barros et al., (2016) destacam a presença de Vinctifer comptoni em folhelhos da Formação Ipubi, indicando que poderia ser um peixe diádromo, vivendo em ambiente marinho e migrando para áreas menos salinas. Além disso, muitos peixes da Formação Romualdo já foram encontrados em formas juvenis na Formação Crato, que possui ambiente com menos salinidade e que, provavelmente, foi usado como um berçário (Leal \& Brito, 2004; Brito \& Yabumoto, 2011).

Os caranguejos braquiúros, assim como os peixes, ocupam quase todos os ambientes aquáticos. A maioria das espécies são marinhas e estuarinas, sendo mais bem representadas no registro fóssil em águas rasas (Jagt et al., 2015; Lee, 2015). Exucarcinus gonzagai é um raninoide, e como tal, seria também uma espécie marinha (Luque, 2015; Prado et al., 2018b). 
Inferir as características ecológicas de Romualdocarcinus salesi, por sua vez, é mais difícil dado às dúvidas quanto ao seu posicionamento nas categorias taxonômicas mais elevadas. Sabe-se que $R$. salesi já foi encontrado associado a Exucarcinus gonzagai em coquinas com equinoides, organismos estenohalinos que demandam altas salinidades para sobrevivência, e moluscos marinhos da Formação Romualdo (Prado et al., 2018b). Além disso, a sua possível espécie-irmã, Eogeryon elegius Ossó, 2016, também foi coletada em uma unidade marinha de plataforma rasa no Cretáceo Superior da Espanha.

Pelas informações levantadas, a associação estudada corresponderia a um ambiente com influência marinha, caracterizando uma fase transicional ou marinha rasa da Formação Romualdo.

\section{Distribuição paleogeográfica}

Vinctifer comptoni e Rhacolepis buccalis são espécies de peixes comuns no Aptiano-Albiano das bacias sedimentares do Nordeste brasileiro, estando presente nas formações Romualdo, Bacia do Araripe (Maisey, 1991; Duque \& Barreto, 2018; Lopes \& Barreto, 2019), Codó, Bacia do Parnaíba (Brito et al., 2016; Lindoso et al., 2016) e, Riachuelo, Bacia de Sergipe (Gallo et al., 2012; Arai, 2014).

No Brasil, o gênero Rhacolepis é representado pelas espécies R. buccalis e R. defiorei D'Erasmo, 1938, esta última limitada à Formação Romualdo e ainda controversa (Voltani, 2016). O gênero Vinctifer é representado pelas espécies Vinctifer longirostris Silva-Santos, 1990, Formação Marizal, Bacia do Tucano (Gallo et al., 2012), Vinctifer punctatus Silva-Santos, 1985, nas formações Maceió e Muribeca, Bacia de Sergipe/Alagoas (Brito, 1997; Souto \& Schwanke, 2010) e Vinctifer comptoni, Formação Romualdo, Bacia do Araripe.

Ambos os gêneros ocorrem em diferentes localidades pelo mundo. Rhacolepis tem distribuição limitada ao Albiano da Colômbia, México e Venezuela (Silva-Santos \& Oliveira, 1994; Maisey, 2000; González-Rodríguez et al., 2016). Vinctifer, com exceção das ocorrências jurássicas no México e Antártida (Arratia et al., 2004; Cantalice et al., 2018), tem distribuição também no Cretáceo, incluindo o Aptiano-Albiano da Colômbia e Albiano-Cenomaniano da Venezuela (Moody \& Maisey, 1994; Schultze \& Stöhr, 1996; Maisey, 2000; González-Rodríguez et al., 2016; Cantalice et al., 2018).

Brito (1997) também relatou a presença do gênero Vinctifer no Albiano da Austrália. No entanto, Moody \& Maisey (1994) já relatavam que a ocorrência proveniente da Austrália era confusa, assim como a ocorrência na Guiné Equatorial. Embora o peixe descrito na Austrália já tenha pertencido aos gêneros Belonostomus (Etheridge \& Woodward, 1892) e Vinctifer (Brito, 1997), atualmente é classificado como Richmondichthys sweeti Bartholomai, 2004 e incluso na família Aspidorhynchidae.

Em relação aos caranguejos, a família Orithopsidae é representada no Aptiano-Albiano dos EUA, Inglaterra e também Colômbia, incluindo a Formação Paja, onde são encontrados os fósseis de Vinctifer, compreendendo os gêneros Planocarcinus, Bellcarcinus e Colombicarcinus, táxons muito semelhantes a Exucarcinus e de mesma família (Luque et al., 2012; Luque, 2014; Karasawa et al., 2014; Schweitzer et al., 2016). Romualdocarcinus salesi, por sua vez, é muito semelhante ao gênero de afinidade tetiana e endêmico ao Cenomaniano da Espanha, Eogeryon (Ossó, 2016; Prado et al., 2018b).

Nota-se que as espécies de peixes e caranguejos aqui estudados possuem uma distribuição restrita ao AptianoAlbiano nas bacias sedimentares do Nordeste. Em estratos correlatos mundialmente, encontram-se os táxons irmãos ocupando o limite do Mar de Tétis (Figura 7), com exceção da possível ocorrência de Vinctifer na Austrália, totalmente dúbia. Isto sugere que a ingressão marinha que teria alcançado as bacias do Nordeste durante o Eocretáceo se deu a partir do antigo Mar de Tetis, possivelmente através da Bacia do Parnaíba, seguindo o modelo de ingressão proposto por Arai (2014). Os dados levantados divergem, portanto, da hipótese de transgressão marinha no Araripe a partir da Bacia Recôncavo-Tucano, por sua vez baseada em paleocorrentes (Assine, 1994; Assine et al., 2014; 2016; Custódio et al., 2017), e sugere que o Mar de Tetis seria uma importante rota de dispersão dos peixes e caranguejos durante o Eocretáceo.

\section{CONSIDERAÇÕES FINAIS}

Os ictiólitos da Formação Romualdo com as espécies Vinctifer comptoni e Rhacolepis buccalis, procedentes de localidades fossilíferas no estado de Pernambuco, apresentam a incomum presença de carapaças dorsais de caranguejos Exucarcinus gonzagai e Romualdocarcinus salesi. Baseado na análise paleocológica e bioestratinômica, esta associação possivelmente não reflete uma interação entre os indivíduos; os restos ou mudas de caranguejos seriam mais antigos que os peixes no sedimento e preservados juntos devido a ação de algum evento não seletivo.

Um pléon isolado com formato subquadrangular próximo a carapaça de Romualdocarcinus salesi preservado no ictiólito corrobora com a hipótese de Prado et al. (2018b), que classificam a espécie como um possível Eubrachyura. Considerando que as espécies Exucarcinus gonzagai e Araripercarcinus ferreirai são raninoides, com apenas a parte dorsal e ventral preservadas, respectivamente, e estão presentes no mesmo nível dos peixes da Formação Romualdo, há uma possibilidade de sinonímia entre elas.

Os dados obtidos com o levantamento paleoecológico e paleogeográfico dos fósseis de peixes e caranguejos aqui apresentados reforçam a hipótese de sedimentação transicional a marinha rasa com influência tetiana no Araripe, uma vez que Vinctifer e Rhacolepis ocorriam no antigo Mar de Tetis durante o Eocretáceo e distribuem-se nas bacias brasileiras seguindo o modelo de seaway proposto por Arai (2014). Ademais, o gênero Vinctifer distribui-se nos mesmos estratos albo-aptianos onde são encontrados os caranguejos oritopsídeos na América do Sul (i.e. Brasil e Colômbia), sugerindo uma possível rota marinha entre estes depósitos. 


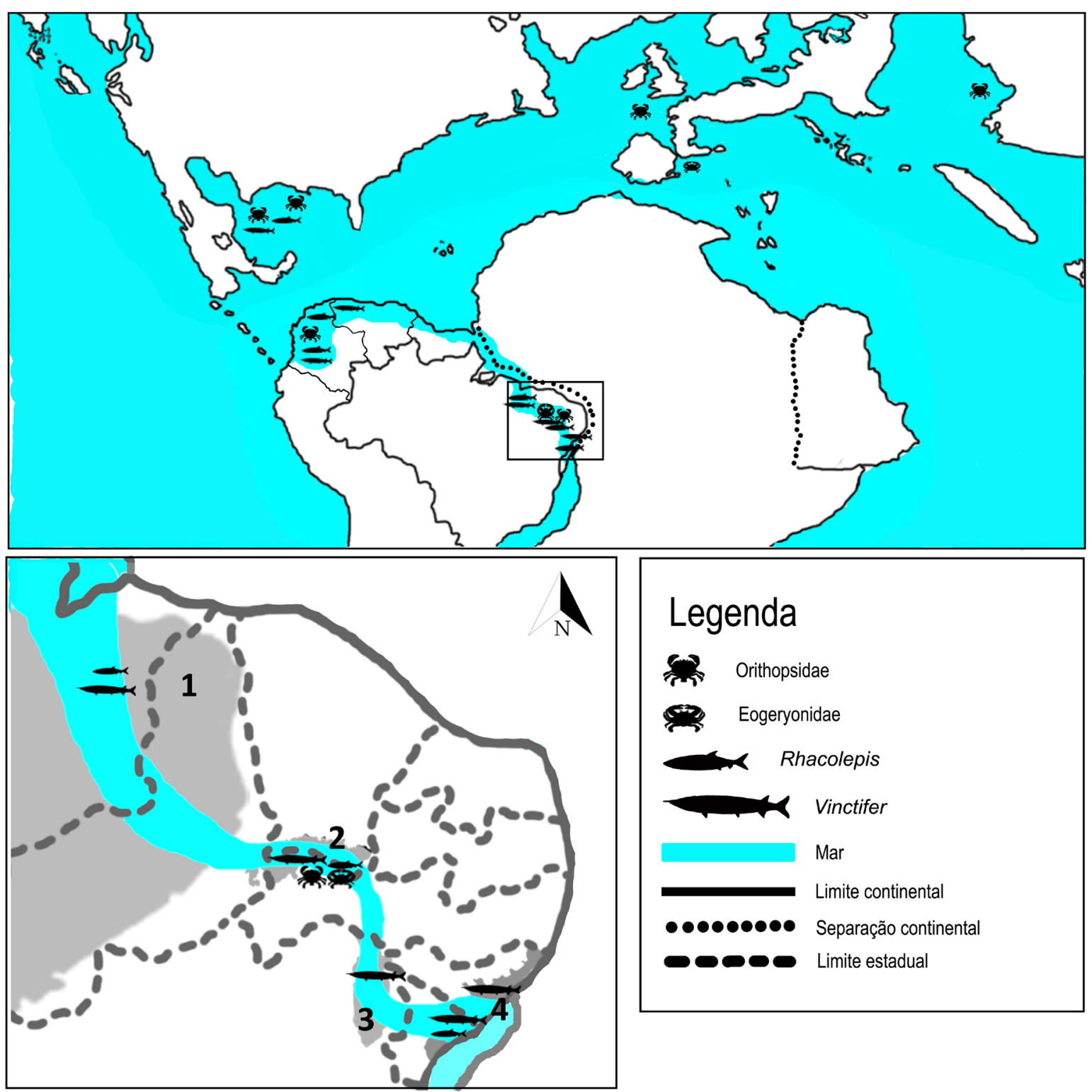

Figura 7. Distribuição paleogeográfica dos peixes dos gêneros Vinctifer e Rhacolepis e caranguejos das famílias Orithopsidae e Eogeryonidae no AptianoAlbiano. Modificado de Scotese (2014) e Schweitzer et al. (2016). Ocorrências brasileiras: 1, Bacia do Parnaíba; 2, Bacia do Araripe; 3, Bacia do Tucano; 4, Bacia de Sergipe/Alagoas.

Figure 7. Paleogeographic distribution of fish of the genera Vinctifer and Rhacolepis and crabs of the families Orithopsidae and Eogeryonidae during the Aptian-Albian. Modified from Scotese (2014) and Schweitzer et al. (2016). Brazilian occurrences: 1, Parnaíba Basin; 2, Araripe Basin; 3, Tucano Basin; 4, Sergipe / Alagoas Basin.

\section{AGRADECIMENTOS}

Os autores agradecem ao técnico do laboratório de Paleontologia do Departamento de Geologia da UFPE, R.R.C. Duque, pela preparação mecânica dos espécimes. A PETROBRAS-ANP, pelo projeto "Análise paleoecológica e bioestratigráfica do Albiano-Aptiano da Bacia do Araripe baseado em microfósseis carbonáticos e palinomorfos", que deu suporte aos trabalhos de campo, a M.C. do Prado Filho pelo auxílio na coleta e aos revisores anônimos pela atenciosa leitura do manuscrito e valiosas sugestões e comentários.

\section{REFERÊNCIAS}

Agassiz, L. 1841. On the fossil fishes found by Mr. Gardner in the province of Ceará, in the north of Brazil. Edinburgh New Philosophical Journal, 30:82-84.

Allison, P.A. 1986. Soft-bodied animals in the fossil record: the role of decay in fragmentation during transport. Geology, 14:979981. doi:10.1130/0091-7613(1986) 14<979:SAITFR >2.0.CO;2

Arai, M. 2014. Aptian/Albian (Early Cretaceous) paleogeography of the South Atlantic: a paleontological perspective. Brazilian Journal of Geology, 44:339-350. doi:10.5327/Z23174889201400020012 
Arai, M. \& Coimbra, J.C. 1990. Análise paleoecológica do registro das primeiras ingressões marinhas na Formação Santana (Cretáceo Inferior da Chapada do Araripe). In: SIMPÓSIO SOBRE A BACIA DO ARARIPE E BACIAS INTERIORES DO NORDESTE, 1, 1990. Atas, Crato, p. 225-239.

Arai, M.; Lana, C.C. \& Pedrão, E. 1994. Ecozona Subtilisphaera spp.: registro eocretáceo de um importante episódio ecológico do Oceano Atlântico primitivo. Acta Geologica Leopoldensia, 39:521-538.

Araripe, R.V.C.; Oliveira, D.H.; Tomé, M.E.T.R.; Mello, R.M.; Barreto, A.M.F. \& Sial, A.N. 2019. Análise isotópica de oxigênio e carbono em microfósseis da Formação Romualdo, Cretáceo Inferior, Bacia do Araripe, Pernambuco, nordeste do Brasil. Geochimica Brasiliensis, 33:133-142. doi:10.21715/GB23582812.2019332133

Arratia, G.; Scasso, R.A. \& Kiessling, W. 2004. Late Jurassic fishes from Longing Gap, Antarctic Peninsula. Journal of Vertebrate Paleontology, 24:41-55. doi:10.1671/1952-4

Assine, M.L. 1994. Paleocorrentes e paleogeografia na Bacia do Araripe, Nordeste do Brasil. Revista Brasileira de Geociências, 24:223-232.

Assine, M.L. 2007. Bacia do Araripe. Boletim de Geociências da Petrobras, 15:371-389.

Assine, M.L.; Perinotto, J.A.J.; Custódio, M.A.; Neumann, V.H.; Varejão, F.G. \& Mescolotti, P.C. 2014. Sequências deposicionais do Andar Alagoas da Bacia do Araripe, Nordeste do Brasil. Boletim de Geociencias da Petrobras, 22:3-28.

Assine, M.L.; Quaglio, F.; Warren, L.V. \& Simões, M.G. 2016. Comments on paper by M. Arai “Aptian/Albian (Early Cretaceous) paleogeography of the South Atlantic: a paleontological perspective". Brazilian Journal of Geology, 46:3-7. doi:10.1590/2317-4889201620150046A

Barros, O.A.; Pontes, A.P.; Batista, M.E.P.; Silva, J.H. \& Saraiva, A.A.F. 2016. Aspectos paleoecológicos da macrofauna dos folhelhos associados à camada de gipsita, Bacia do Araripe. Estudos Geológicos, 26:147-156. doi:10.18190/1980-8208/ estudosgeologicos.v26n2p147-156

Bartholomai, A. 2004. The large aspidorhynchid fish, Richmodichthys sweeti (Etheridge Jnr and Smith Woodward, 1891) from Albian marine deposits of Queensland, Australia. Memoirs of the Queensland Museum, 49:521-536.

Bemvenuti, M.D.A. \& Fischer, L.G. 2010. Peixes: morfologia e adaptações. Cadernos de Ecologia Aquática, 5:31-54.

Beurlen, K. 1963. Geologia e estratigrafia da Chapada do Araripe. In: CONGRESSO NACIONAL DE GEOLOGIA, 17, 1963. Anais, Recife, SBG/SUDENE. p. 1-47.

Beurlen, K. 1964. As espécies dos Cassiopinae, nova subfamilia dos Turritellidae, no Cretáceo do Brasil. Arquivos de GeologiaUFPE, 5:1-44.

Beurlen, K. 1966. Novos equinóides no Cretáceo do Nordeste do Brasil. Anais da Academia Brasileira de Ciências, 38:455-464.

Bishop, G.A. 1972a. Crab bitten by a fish from the upper Cretaceous Pierre Shale of South Dakota. Geological Society of America Bulletin, 83:3823-3825. doi:10.1130/00167606(1972)83[3823:CBBAFF]2.0.CO;2

Bishop, G.A. 1972b. Moults of Dakoticancer overanus, an Upper Cretaceous crab from the Pierre shale of South Dakota. Palaeontology, 15:631-636.

Bishop, G.A. 1978. Two new crabs, Sodakus tatankayotankaensis n. gen., n. sp. and Raninella oaheensis n. sp. (Crustacea, Decapoda), from the Upper Cretaceous Pierre Shale of South Dakota. Journal of Paleontology, 52:608-617.
Bishop, G.A. 1986. Taphonomy of the North American Decapods. Journal of Crustacean Biology, 6:326-355. doi:10.1163/193724086X00190

Brito, P.M. 1997. Révision des Aspidorhynchidae (Pisces, Actinopterygii) du Mésozoïque: ostéologie, relations phylogénétiques, données environnementales et biogéographiques. Geodiversitas, 19:681-772.

Brito, P.M.; Leal, M.E.C. \& Gallo, V. 2013. A new lower Cretaceous guitarfish (Chondrichthyes, Batoidea) from the Santana formation, Northeastern Brazil. Boletim do Museu Nacional, Geologia, 75:1-13.

Brito, P.M.; Lindoso, R.M.; Carvalho, I.S. \& Machado, G.P. 2016. Discovery of $\dagger$ Obaichthyidae gars (Holostei, Ginglymodi, Lepisosteiformes) in the Aptian Codó Formation of the Parnaíba Basin: remarks on paleobiogeographical and temporal range. Cretaceous Research, 59:10-17. doi:10.1016/j. cretres.2015.10.017

Brito, P.M. \& Yabumoto, Y. 2011. An updated review of the fish faunas from the Crato and Santana formations in Brazil, a close relationship to the Tethys fauna. Bulletin of the Kitakyushu Museum of Natural History and Human History, 9:107-136

Bruno, A.P. \& Hessel, M.P. 2006. Registros paleontológicos do Cretáceo marinho na Bacia do Araripe. Estudos Geológicos, 16:30-49.

Cantalice, K.M.; Alvarado-Ortega, J. \& Brito, P.M. 2018. On the occurrence of Vinctifer ferrusquiai sp. nov. (Actinopterygii, Aspidorhynchiformes) in the Kimmeridgian (Late Jurassic) deposits near Tlaxiaco, Oaxaca, southern Mexico. Revista Mexicana de Ciencias Geológicas, 35:179-187. doi:10.22201/ cgeo.20072902e.2018.2.713

Costa, A.C.; Junior, L.F.S.; Domingos, F.F.T. \& Fonseca, M.L. 2005. Alimentação da pirambeba Serrasalmus spilopleura Kner, 1858 (Characidae; Serrasalminae) em um reservatório do sudeste brasileiro. Acta Scientiarum. Biological Sciences, 27:365-369. doi:10.4025/actascibiolsci.v27i4.1331

Custódio, M.A.; Quaglio, F.; Warren, L.V.; Simões, M.G.; Fürsich, F.T.; Perinotto, J.A.J. \& Assine, M.A. 2017. The transgressiveregressive cycle of the Romualdo Formation (Araripe Basin): sedimentary archive of the Early Cretaceous marine ingression in the interior of Northeast Brazil. Sedimentary Geology, 35:1-15. doi:10.1016/j.sedgeo.2017.07.010

D’Erasmo, G. 1938. Ittioliti Cretacei del Brasile. Atti della Reale Accademie dele Scienze Fisiche e Matematiche, 1:1-44.

Duque, R.R.C. \& Barreto, A.M.F. 2018. Os vertebrados fósseis da Formação Romualdo, (Cretáceo Inferior, Bacia do Araripe) em Exu e Araripina, Pernambuco, Nordeste do Brasil. Anuário do Instituto de Geociências - UFRJ, 41:5-14. doi:10.11137/2018_1_05_14

Etheridge, R. \& Woodward, A.S. 1892. On the occurrence of the genus Belonostomus in the Rolling Downs Formation (Cretaceous) of central Queensland: Melbourne. Transactions of the Royal Society of Victoria, 2:1-7.

Gaffney, E.S.; Almeida Campos, D. \& Hirayama, R. 2001. Cearachelys, a new side-necked turtle (Pelomedusoides: Bothremydidae) from the Early Cretaceous of Brazil. American Museum Novitates, 3319:1-20. doi:10.1206/00030082(2001)319<0001:CANSNT>2.0.CO;2

Gallo, V.; Figueiredo, F.J. \& Carvalho, M.S.S. 2012. Síntese da paleoictiofauna marinha das bacias costeiras do Brasil e comentários sobre a paleoictiofauna cronocorrelata da margem oeste da África. In: V. Gallo; H.M.A. Silva; P.M. Brito \& F. 
Figueiredo (eds.) Paleontologia de Vertebrados: relações entre América do Sul e África, Editora Interciência, p. 175-206.

González-Rodríguez, K.A.; Fielitz, C.; Bravo-Cuevas, V.M. \& Baños-Rodríguez, R.E. 2016. Cretaceous osteichthyan fish assemblages from Mexico. New Mexico Museum of Natural History and Science Bulletin, 71:1-14.

Guinot, D.; De Angeli, A. \& Garassino, A. 2008. Marocarcinidae, a new eubrachyuran family, and Marocarcinus pasinii $\mathrm{n}$. gen., $\mathrm{n}$. sp. from the Upper Cretaceous (Cenomanian-Turonian) of Gara Sbaa, southeastern Morocco (Crustacea, Decapoda, Brachyura). Atti della Società Italiana di Scienze Naturali e del Museo Civico di Storia Naturale in Milano, 149:25-36.

Guinot, D.; Vega, F.J. \& Van Bakel, B. 2008. Cenomanocarcinidae n. fam., a new Cretaceous podotreme family (Crustacea, Decapoda, Brachyura, Raninoidia), with comments on related families. Geodiversitas, 30:681-719.

Jagt, J.W.M.; van Bakel, B.W.M; Guinot, D.; Fraaije, R.H.B. \& Artal, P. 2015. Fossil Brachyura. In: P. Castro; P. Davie; D. Guinot; F. Schram \& C.V. Klein (eds.) Treatise on Zoology - Anatomy, Taxonomy, Biology, The Crustacea, 9C, Brill, p. 847-920.

Jordan, D.S. \& Branner, J.C. 1908. The Cretaceous fishes of Ceará, Brazil. Smithsonian Miscellaneous Collections, 52:1-29.

Karasawa, H.; Schweitzer, C.E.; Feldmann, R.M. \& Luque, J. 2014. Phylogeny and Classification of the Raninoida (Crustacea: Brachyura). Journal of Crustacean Biology, 34:216-272. doi:10.1163/1937240X-00002216

Kellner, A.W.A. 2002. Membro Romualdo da Formação Santana, Chapada do Araripe, CE - Um dos mais importantes depósitos fossilíferos do Cretáceo brasileiro. In: C. Schobbenhaus; D.A. Campos; E.T. Queiroz; M. Winge \& M.L.C. Berbetborn (eds.) Sitios Geológicos e Paleontológicos do Brasil, DNPM/CPRM - Comissão Brasileira de Sítios Geológicos e Paleontológicos (SIGEP), p. 121-130.

Klompmaker, A.A.; Chistoserdov, A.Y. \& Felder, D.L. 2016. Possible shell disease in 100 million-year-old crabs. Diseases of aquatic organisms, 119:91-99. doi:10.3354/dao02988

Klompmaker, A.A.; Karasawa, H.; Portell, R.W.; Fraaije, R.H.B. \& Ando, Y. 2013. An overview of predation evidence found on fossil decapod crustaceans with new examples of drill holes attributed to gastropods and octopods. Palaios, 28:599-613. doi:10.2110/palo.2013.p13-026r

Leal, M.E.C \& Brito, P.M. 2004. The ichthyodectiform Cladocyclus gardneri (Actinopterygii: Teleostei) from the Crato and Santana Formations, Lower Cretaceous of Araripe Basin, North-Eastern Brazil. Annales de Paléontologie, 90:103-113. doi:10.1016/j. annpal.2004.01.001

Lee, S.Y. 2015. Ecology of Brachyura. In: P. Castro; P. Davie; D. Guinot; F. Schram \& C.V. Klein (eds.) Treatise on Zoology - Anatomy, Taxonomy, Biology, The Crustacea, 9C, Brill, p. 469-541.

Lima, M.R. 1978. Palinologia da Formação Santana (Cretáceo do Nordeste). Instituto de Geociências, Universidade de São Paulo, Ph.D. thesis, $337 \mathrm{p}$.

Lima, F.J.; Saraiva, A.A.F. \& Sayão, J.M. 2012. Revisão da paleoflora das Formações Missão Velha, Crato e Romualdo, Bacia do Araripe, Nordeste do Brasil. Estudos Geológicos, 22:99-115. doi:10.18190/1980-8208/estudosgeologicos.v22n1p99-115

Lindoso, R.M.; Maisey, J.G. \& Carvalho, I.S. 2016. Ichthyofauna from the Codó Formation, Lower Cretaceous (Aptian, Parnaíba Basin), Northeastern Brazil and their paleobiogeographical and paleoecological significance. Palaeogeography, Palaeoclimatology, Palaeoecology, 447:53-64. doi:10.1016/j. palaeo.2016.01.045
Lopes, G.L.B. \& Barreto, A.M.F.A. 2019. Paleoictiofauna da Formação Romualdo, Cretáceo Inferior, da Bacia Sedimentar do Araripe, Pernambuco, Nordeste do Brasil. Anuário do Instituto de Geociências - UFRJ, 42:396-409. doi:10.11137/2019_4_396_409

Luque, J. 2014. A new genus and species of raninoidan crab (Decapoda, Brachyura) from the Lower Cretaceous of Colombia, South America. Scripta Geologica, 147:27-34.

Luque, J. 2015. A puzzling frog crab (Crustacea: Decapoda: Brachyura) from the Early Cretaceous Santana Group of Brazil. Frog first or crab first? Journal of Systematic Palaeontology, 13:153-166. doi:10.1080/14772019.2013.871586

Luque, J.; Feldmann, R.M.; Schweitzer, C.E.; Jaramillo, C. \& Cameron, C.B. 2012. The oldest frog crabs (Decapoda: Brachyura: Raninoida) from the Aptian of northern South America. Journal of Crustacean Biology, 32:405-420. doi:10.1163/193724012X626539

Mabesoone, J.M. \& Tinoco, I.M. 1973. Paleoecology of the Aptian Santana Formation (Northeastern Brazil). Palaeogeography, Palaeclimatology, Palaeoecology, 14:97-118. doi:10.1016/00310182(73)90006-0

Mabesoone, J.M.; Viana, M.S.S. \& Lima-Filho, M.F. 1999. Late Mesozoic history of sedimentary basins in NE Brazilian Borborema Province before the final separation of South America and Africa. 3: Paleogeogeoghraphy. In: SIMPÓSIO SOBRE O CRETÁCEO DO BRASIL, 5, 1999. Boletim de Resumos, Serra Negra, p. 621-626.

Maisey, J.G. 1991. Santana Fossil: an illustrated atlas. New Jersey, Tropical Fish Hobbyist Publications, 459 p.

Maisey, J.G. 1994. Predator-prey relationships and trophic level reconstruction in a fossil fish community. Environmental Biology of Fishes, 40:1-22. doi:10.1007/BF00002179

Maisey, J.G. 2000. Continental break up and the distribution of fishes of Western Gondwana during the Early Cretaceous. Cretaceous Research, 21:281-314. doi:10.1006/cres.1999.0195

Maisey, J.G. \& Carvalho, G.P. 1995. First records of fossil sergestid decapods and fossil brachyuran crab larvae (Arthropoda, Crustacea), with remarks on some supposed palaemonid fossils, from the Santana Formation (Aptian-Albian, NE Brazil). American Museum Novitates, 3132:1-17.

Marques, F.O.; Nogueira, F.C.C.; Bezerra, F.H.R. \& Castro, D.L. 2014. The Araripe Basin in NE Brazil: an intracontinental graben inverted to a high-standing horst. Tectonophysics, 630:251-264. doi:10.1016/j.tecto.2014.05.029

Martill, D.M. 1988. The preservation of fossil fishes in concretions from the Cretaceous of Brazil. Palaeontology, 31:1-18.

Martill, D.M. 1989. The Medusa effect: instantaneous fossilization. Geology Today, 201-205.doi:10.1111/j.1365-2451.1989.tb00671.x

Martill, D.M.; Brito, P.M. \& Washington-Evans, C. 2008. Mass mortality of fishes in the Santana Formation (Lower Cretaceous, ?Albian) of northeast Brazil. Cretaceous Research, 29:649-658. doi:10.1016/j.cretres.2008.01.012

Martins-Neto, R.G. 1987. Primeiro registro de decápode na Formação Santana, Bacia do Araripe (Cretáceo Inferior), Brasil. Ciência e Cultura, 39:406-410.

Matos, R.M.D. 1992. The northeast Brazilian rift system. Tectonics, 11:766-791. doi:10.1029/91TC03092

Melo, R.B.; Guzmán, J.; Almeida Lima, D.; Piosevan, E.K.; Neumann, V.H.M.L. \& Sousa, A.J. 2020. New marine data and age accuracy of the Romualdo formation, Araripe Basin, Brazil. Scientific Reports, 10:15779. doi:10.1038/s41598-020-72789-8

Milne-Edwards, A. \& Bouvier, E.L. 1923. Reports on the results of dredging under the supervision of Alexander Agassiz in the 
Gulf of Mexico (1877-78), in the Caribbean Sea (1878-79), and along the Atlantic coast of the United States (1880) by the U. S. Coast Survey Steamer "Blake". XLVII. Les porcellanidés et les brachyures. Memoirs of the Museum of Comparative Zoology at Harvard College, 47:283-395.

Moody, J.M. \& Maisey, J.G. 1994. New Cretaceous marine vertebrate assemblages from north-western Venezuela and their significance. Journal of Vertebrate Paleontology, 14:1-8.

Neumann, V.H. \& Cabrera, L. 1999. Una nueva propuesta estratigráfica para la tectono secuencia post-rifte de la cuenca de Araripe, Noreste de Brasil. In: SIMPÓSIO SOBRE O CRETÁCEO BRASILEIRO, 5, 1999. Boletim de Resumos, Serra Negra, p. 279-285.

Oliveira, G.R. 2007. Aspectos tafonômicos de Testudines da Formação Santana (Cretáceo Inferior), Bacia do Araripe, Nordeste do Brasil. Anuário do Instituto de Geociências - UFRJ, 30:83-93. doi:10.11137/2007_1_83-93

Ossó, À. 2016. Eogeryon elegius n. gen. and n. sp. (Decapoda: Eubrachyura: Portunoidea), one of the oldest modern crabs from late Cenomanian of the Iberian Peninsula. Boletín de la Sociedad Geológica Mexicana, 68:231-246.

Pereira, P.A.; Cassab, R.C.T. \& Barreto, A.M.F. 2017. Paleoecologia e paleogeografia dos moluscos e equinoides da Formação Romualdo, Aptiano-Albiano da Bacia do Araripe, Brasil. Anuário do Instituto de Geociências-UFRJ, 40:180-198. doi:10.11137/2017 2 180 198

Pinheiro, A.P.; Saraiva, A.Á.F. \& Santana, W. 2014. Shrimps from the Santana Group (Cretaceous: Albian): new species (Crustacea: Decapoda: Dendrobranchiata) and new record (Crustacea: Decapoda: Caridea). Anais da Academia Brasileira de Ciências, 86:663-670. doi:10.1590/0001-3765201420130338

Plotnick, R.E.1986. Taphonomy of a modern shrimp: implications for the arthropod fossil record. Palaios, 1:286-293. doi: $10.2307 / 3514691$

Polck, M.A.R.; Carvalho, M.S.S.; Miguel, R. \& Gallo, V. 2015. Guia de identificação de peixes fósseis das formações Crato e Santana da Bacia do Araripe. Rio de Janeiro, CPRM, 69 p.

Pompeu, P.S. 1999. Dieta da pirambeba Serrasalmus brandtii Reinhardt (Teleostei, Characidae) em quatro lagoas marginais do rio São Francisco, Brasil. Revista Brasileira de Zoologia, 16:19-26. doi:10.1590/S0101-81751999000600003

Ponte, F.C. \& Ponte Filho, F.C. 1996. Evolução tectônica e classificação da Bacia do Araripe. In: SIMPÓSIO SOBRE O CRETÁCEO DO BRASIL, 4, 1996. Boletim de Resumos, Rio Claro, p. 123-133.

Poulicek, M.; Goffinet, G.; Jeuniaux, C. \& Gooday, G.W. 1988. Early digenesis of skeletal remains in marine sediments: a ten years study. Bulletin de la Société Royale des Sciences de Liège, 57:313-330.

Prado, L.A.C.; Fambrini, G.L. \& Barreto, A.M.F. 2018a. Tafonomy of macroinvertebrates and Albian marine ingression as recorded by the Romualdo Formation (Cretaceous, Araripe Basin, Brazil). Brazilian Journal of Geology, 48:519-531. doi:10.1590/23174889201820180048

Prado, L.A.C.; Luque, J.; Barreto, A.M.F. \& Palmer, R. 2018b. New orithopsid crabs from the Aptian-Albian Romualdo Formation, Santana Group of Brazil: evidence for a tethyan connection to the Araripe Basin. Acta Palaeontologica Polonica, 63:737-750. doi:10.4202/app.00480.2018

Prado, L.A.C.; Pereira, P.A.; Sales, A.M.F. \& Barreto, A.M.F. 2015. Taphonomic and paleoenvironmental considerations for the concentrations of macroinvertebrate fossils in the Romualdo Member, Santana Formation, Late Aptian-Early Albian, Araripe
Basin, Araripina, NE, Brazil. Journal of South American Earth Sciences, 62:218-228. doi:10.1016/j.jsames.2015.06.005

Price, L.I. 1973. Quelônio Amphichelydia no Cretáceo Inferior do Nordeste do Brasil. Revista Brasileira de Geociências, 3:84-96

Schultze, H.P. \& Stöhr, D. 1996. Vinctifer (Pisces, Aspidorhynchidae) aus der Unterkreide (oberes Aptium) von Kolumbien: Stuttgart. Neues Jahrbuch für Geologie und Paläontologie, Abhandlungen, 199:395-415.

Schweitzer, C.E.; Feldmann, R.M. \& Lamanna, M.C. 2012. New genus of crab (Brachyura: Raninoida: Necrocarcinidae) from the Upper Cretaceous of West Antarctica, with description of a new species. Annals of Carnegie Museum, 80:147-158. doi:10.2992/007.080.0203

Schweitzer, C.E.; Karasawa, H.; Luque, J. \& Feldmann, R.M. 2016. Phylogeny and classification of Necrocarcinoidea Föster, 1968 (Brachyura:Raninoida) with the description of two new genera. Journal of Crustacean Biology, 36:338-372. doi:10.1163/1937240X-00002432

Scotese, C.R. 2014. Atlas of Early Cretaceous paleogeographic maps. PALEOMAP Atlas for ArcGIS, Mollweide Projection, PALEOMAP Project, Evanston, IL, 2, Maps 23-31.

Silva-Santos, R. 1985. Sobre a presença de Vinctifer Jordan (Pisces, Aspidorhynchiformes) na Formação Muribeca, Estado de Alagoas. Rio de Janeiro, Departamento Nacional da Produção Mineral (Coletânea de Trabalhos Paleontológicos), p. 147-150.

Silva-Santos, R. 1990. Vinctifer longirostris, do Cretáceo Inferior da formação Marizal, Estado da Bahia, Brasil. Anais da Academia Brasileira de Ciências, 62:251-260.

Silva-Santos, R. \& Oliveira, A.R. 1994. Rhacolepis buccalis Agassiz, 1841 (Pisces, Elopomorpha), no Cretáceo Inferior da Colômbia, AS. Anais Academia Brasileira de Ciências, 66:167-171.

Silva-Santos, R. \& Valença, J.G. 1968. A Formação Santana e sua paleoictiofauna. Anais da Academia Brasileira Ciências, 40:339-360.

Souto, P.R.F. \& Schwanke, C. 2010. Ocorrência de coprólitos de vertebrados na Bacia de Alagoas, Cretáceo Inferior. Boletim do Museu Paraense Emílio Goeldi Ciências Naturais, 5:189-194.

Stempien, J.A. 2005. Brachyuran taphonomy in a modern tidalflat environment: preservation potential and anatomical bias. Palaios, 20:400-410. doi:10.2110/palo.2004.p04-40

Valença, L.M.M.; Neumann, V.H. \& Mabesoone, J.M. 2003. An overview on Callovian - Cenomanian intracratonic basins of Northeast Brazil: onshore stratigraphic record of the opening of the Southern Atlantic. Geologica Acta, 1:261-275. doi:10.1344/105.000001614

Voltani, C.G. 2016. Redescrição osteológica comentada e aspectos paleoecológicos dos gêneros Notelops $e$ Rhacolepis (Pachyrhizodontoidei). Programa de Pós-Graduação em Geociências e Meio Ambiente, Universidade de São Paulo, Ph.D. thesis, $178 \mathrm{p}$.

Wilby, P.R. \& Martill, D.M. 1992. Fossil fish stomachs: a microenvironment for exceptional preservation. Historical Biology, 6:25-36. doi:10.1080/10292389209380416

Received in 17 November, 2020; accepted in 01 March, 2021. 